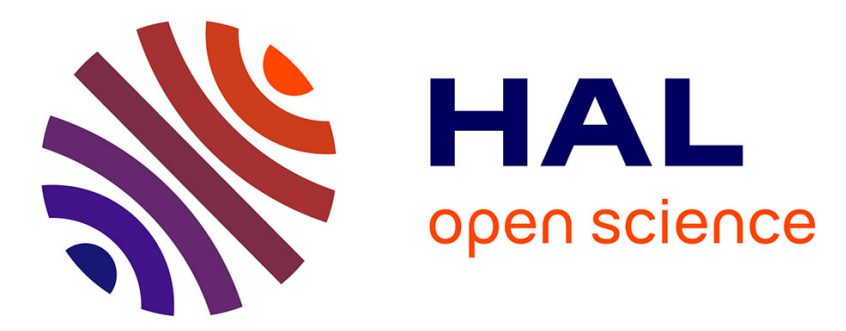

\title{
Low-Temperature Mechanical Loss Spectroscopy of 5N Lead
}

O. Progin, G. Gremaud, W. Benoit

\section{To cite this version:}

O. Progin, G. Gremaud, W. Benoit. Low-Temperature Mechanical Loss Spectroscopy of 5N Lead. Journal de Physique IV Proceedings, 1996, 06 (C8), pp.C8-183-C8-186. 10.1051/jp4:1996837 . jpa00254645

\section{HAL Id: jpa-00254645 https://hal.science/jpa-00254645}

Submitted on 1 Jan 1996

HAL is a multi-disciplinary open access archive for the deposit and dissemination of scientific research documents, whether they are published or not. The documents may come from teaching and research institutions in France or abroad, or from public or private research centers.
L'archive ouverte pluridisciplinaire HAL, est destinée au dépôt et à la diffusion de documents scientifiques de niveau recherche, publiés ou non, émanant des établissements d'enseignement et de recherche français ou étrangers, des laboratoires publics ou privés. 
JOURNAL DE PHYSIQUE IV

Colloque C8, supplément au Journal de Physique III, Volume 6, décembre 1996

\title{
Low-Temperature Mechanical Loss Spectroscopy of $5 \mathrm{~N}$ Lead
}

\author{
O. Progin, G. Gremaud and W. Benoit \\ Ecole Polytechnique Fédérale de Lausanne, Département de Physique, Institut de Génie Atomique, \\ 1015 Lausanne, Switzerland
}

\begin{abstract}
By internal friction measurements in $5 \mathrm{~N}$ lead, a relaxation peak is observed in the vicinity of $40 \mathrm{~K}$ at a frequency of $250 \mathrm{~Hz}$, and in the vicinity of $150 \mathrm{~K}$ at a frequency of $18 \mathrm{MHz}$ (ultrasonic attenuation). This thermally activated relaxation demonstrates all the characteristics of a Bordoni peak. Using the ultrasonic low-frequency coupling method, new high-temperature signatures were observed on each temperature side of the high frequency Bordoni peak $(150 \mathrm{~K})$. These signatures can only be interpreted if one assumes that a thermally activated kink pair formation (KPF) mechanism is responsible for the high-frequency Bordoni peak. Low-frequency internal friction measurements performed after low-temperature plastic deformation have shown that a modulus softening effect, similar to the softening already observed in aluminium after plastic deformation or after electron irradiation, occurs in lead. This result confirms the existence of the dislocation lubrication process recently proposed to explain the discrepancy between the low-temperature behaviour of the critical resolved shear stress in FCC metals and mechanical spectroscopy measurements, and the signatures observed at very low temperatures suggest that a shortcircuited KPF mechanism is responsible for this lubrication process.
\end{abstract}

\section{INTRODUCTION}

In FCC metals, the Bordoni peaks appearing in the low temperature range are generally considered as intrinsic dislocation relaxations, attributed to the kink pair formation (KPF) mechanism on the $60^{\circ}$ dislocations and on the screw dislocations respectively [1-3]. However, this explanation is controversial, because the Peierls stress that is deduced from the activation energy of the Bordoni relaxation, is very much higher than the Peierls stress obtained from yield stress measurements. As a consequence, the interpretation of the Bordoni relaxation by a KPF mechanism is apparently in complete contradiction with the strength experiments. That is often referred to as the unsolved problem of the Peierls potential in fcc metals.

In order to verify the KPF origin of the Bordoni relaxation, two mechanical spectroscopy methods have been used. These experimental techniques will be presented briefly in Sect. 2 of this paper. In Sect. 3, we will first show that the relaxation peak observed in lead is the Bordoni peak. Then we will attempt to demonstrate that the Bordoni peak is due to a thermally activated kink pair formation. Finally, we will prove the existence of the dislocation lubrication process, due to a short circuit mechanism of the KPF, recently proposed [14-15] to explain the unsolved problem of the Peierls potential in fcc metals.

\section{EXPERIMENTAL TECHNIQUES}

In order to study the dynamic properties of dislocations in lead after a low-temperature plastic deformation, two mechanical spectroscopy methods at medium- and high-frequency have been used (100 $\mathrm{Hz}-20 \mathrm{MHz})$. The samples were prepared from high purity mono- $(99.999 \%)$ and poly-crystalline $(99.9999 \%)$ rods. After the shaping, these beams were annealed 4 hours in a high-vacuum furnace at a temperature of $540 \mathrm{~K}$.

In the medium frequency range $(100 \mathrm{~Hz}-1 \mathrm{kHz})$, the internal friction and the resonance frequency were obtained by a flexural vibration technique of thin clamped beams. The damping of the free vibrations was measured using a discrete Fourier transform method. The vibrations of the sample were electrostatically excited by an electrode in a vacuum of $10^{-6} \mathrm{mbar}$ and detection was realized using the same electrode. In order to reduce parasitic interactions with the sample holder, a particular sample shape is used [4]. This technique leads to a deformation measurement amplitude between $10^{-7}$ and $10^{-5}$. The temperature was varied between $20 \mathrm{~K}$ and $350 \mathrm{~K}$.

The coupling technique is a two-wave acoustic method for the study of the dislocation dynamics [5]. The principle of the ultrasound low-frequency vibration coupling method (US-LF) consists of measuring the attenuation $\alpha$ and the velocity $\mathrm{v}$ of ultrasonic waves in a sample subjected to a permanent sinusoidal low frequency applied stress $\sigma$. The closed curves $\Delta \alpha(\sigma)$ and $\Delta v(\sigma)$, measured during one cycle of the low 
frequency stress, are drawn for different temperatures, maximum amplitudes of applied stress or times. The shapes of the curves $\Delta \alpha(\sigma)$ and $\Delta v / v(\sigma)$ and their time evolution are characteristic for each mechanism controlling the dislocation motion. For this reason, these curves have been called signatures of the dislocation motion mechanism. This technique allows one to measure automatically the attenuation and the relative change in propagation time of longitudinal ultrasonic waves with a sensitivity of $10^{-4} \mathrm{~dB} / \mathrm{ms}$ and $10^{-6}$ respectively, between $4 \mathrm{~K}$ and $300 \mathrm{~K}$. A mechanical system is able to generate a compressive stress of any waveform to the sample between $10^{-4}$ and $10^{-1} \mathrm{~Hz}$ with amplitudes from 0.1 to $50 \mathrm{MPa}$.

\section{RESULTS AND DISCUSSION}

\subsection{The Bordoni relaxation in lead}

Measurements of $5 \mathrm{~N}$ and $6 \mathrm{~N}$ lead were performed using the flexural vibration technique with a deformation amplitude of $10^{-7}$ and a linear increase of temperature at a rate of $1 \mathrm{~K} \mathrm{m^{-1 }}$. Figure 1 displays the internal friction (IF) spectra as a function of the temperature $\mathrm{T}$ in $5 \mathrm{~N}$ lead measured at $250 \mathrm{~Hz}$, in the reference state (4 hours annealing at 540 $\mathrm{K})$ and for different annealing temperatures after cold working. A relaxation peak and a modulus defect (not represented here) appear at about $40 \mathrm{~K}$. For an annealing temperature of $200 \mathrm{~K}$, one obtains the highest relaxation peak and the greatest modulus defect. For higher annealing temperatures, both the relaxation peak height and the modulus defect decrease.

Internal friction measurements of $5 \mathrm{~N}$ lead were also performed using an ultrasonic wave technique. Figure 2 presents the attenuation spectrum in lead as function of temperature

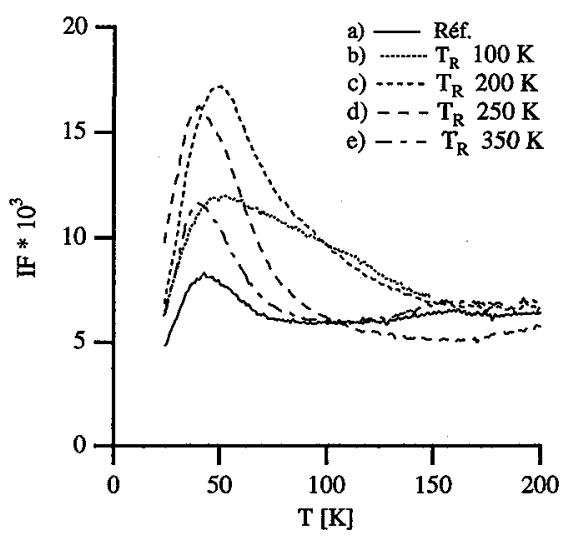

Figure 1: Internal friction measurements as function of temperature in lead $5 \mathrm{~N}$ in the reference state (curve a), after a cold working of $2.7 \%$ at $24 \mathrm{~K}$ and different annealing temperatures (curves b-e).

for an ultrasonic stress frequency of $18.5 \mathrm{MHz}$. This result shows a relaxation peak at $150 \mathrm{~K}$ after a cold working at $4 \mathrm{~K}$ and an annealing at $300 \mathrm{~K}$. It is remarkable because the absolute attenuation, measured by an ultrasonic technique, shows a relaxation peak generated by the high frequency stress.

When the logarithm of the measurement frequency is plotted as a function of the inverse of the relaxation peak temperature (Arrhenius plot), a straight line is obtained (Fig. 3) with our own measurements and those of Wagner [17]. This linear behaviour, in a large frequency range $(1 \mathrm{~Hz}$ to $20 \mathrm{MHz})$, proves that this relaxation peak is thermally activated. From the Arrhenius plot, the following activation energy $\mathrm{E}_{\mathrm{a}}$ and preexponential factor $f_{o}$ are deduced: $E_{a}=0.043 \pm 0.002 \mathrm{eV}$ and $f_{0}=3 \times 10^{8} \pm 2 \times 10^{8} \mathrm{~Hz}$.

Finally, the overall observed characteristics of this relaxation peak correspond in fact to the primary and secondary characteristics generally associated with the Bordoni peak observed in fcc metals [6-9]. We can then conclude that the relaxation peak observed in lead at low and high frequencies is the Bordoni peak.

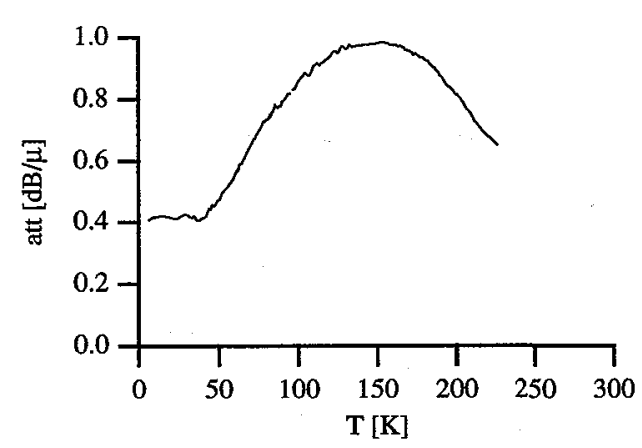

Figure 2: Ultrasonic attenuation as function of the temperature in lead $5 \mathrm{~N}$ measured after a cold working of $0.9 \%$ at $4 \mathrm{~K}$ and an annealing at room temperature .

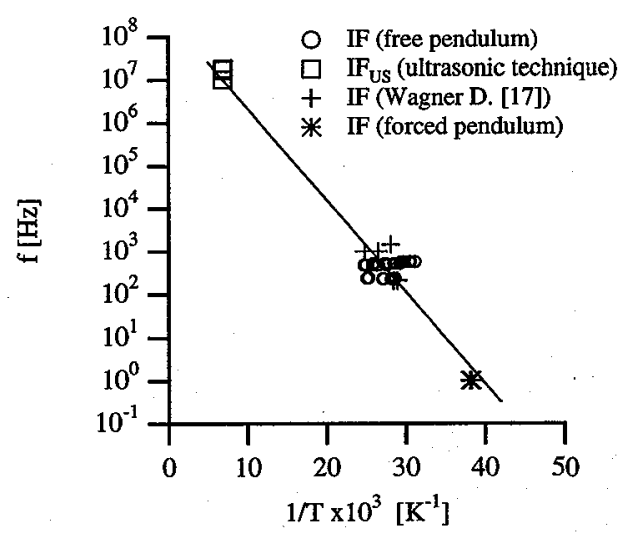

Figure 3: Arrhenius plot. This figure is obtained with the experimental data of this work and those found in literature. 


\subsection{The Bordoni relaxation and the KPF mechanism}

To study the mechanisms responsible for the Bordoni relaxation, signature measurements were performed using the US-LF coupling method.

New high-temperature signatures (Fig. $4 \mathrm{~A}$ ) were obtained on each temperature side of the high frequency (HF) Bordoni peak (150K). The shapes of these signatures are almost parallelograms. But, below and above the high frequency Bordoni peak, the signatures turn clockwise and anti-clockwise respectively, which leads to a kind of "mirror-symmetry" with regard to the HF-Bordoni peak temperature. These signatures are correlated with a non-negligible (hysteretic and athermal) internal friction background observed on the low-frequency IF spectrum in cold worked and annealed lead [10]. Both low frequency internal friction background and correlated signature shapes (parallelograms) were attributed to an athermal dislocation-point defect interaction [18].

If it is assumed that a KPF mechanism is responsible for the HF-Bordoni peak, this KPF mechanism must also be responsible for the "mirror symmetry" of the signatures. Indeed, the dislocation vibration generated by the low-amplitude ultrasonic stress is described by a geometrical kink chain model below the HF-Bordoni peak temperature, and by a classical string model above the HF-Bordoni peak temperature [18]. This means that the ultrasonic stress field can only induce small displacements of the geometrical

a)

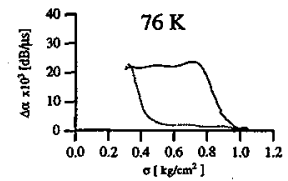

b)

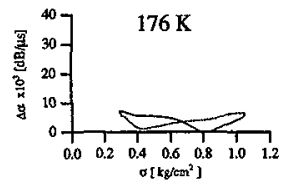

c)

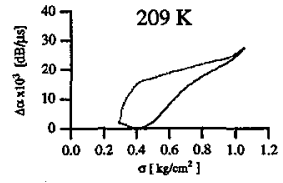

(A)

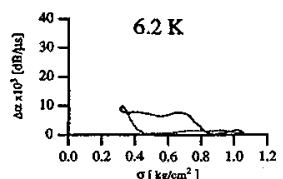

$\sigma\left[\mathrm{kg}_{\mathrm{g}} / \mathrm{cm}^{2}\right]$
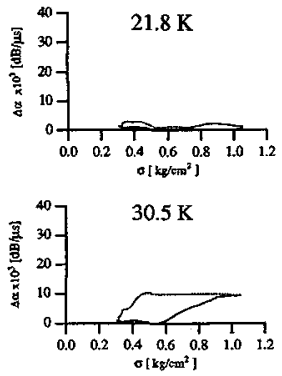

(B)

Figure 4: A) Signatures measured in lead at $76 \mathrm{~K}(\mathrm{a})$, $176 \mathrm{~K}(\mathrm{~b})$ and $209 \mathrm{~K}(\mathrm{c})$. The experimental signatures have been obtained after a cold working of $0.9 \%$ at $4 \mathrm{~K}$ and a room temperature annealing. The arrows upon the signatures shows the course wise.

B) Similar evolution of signatures measured in lead at $6.2 \mathrm{~K}(\mathrm{a}), 21.8 \mathrm{~K}(\mathrm{~b})$ and $30.5 \mathrm{~K}(\mathrm{c})$. decreases if the average displacement of the dislocation due to the low-frequency applied stress increases (hardening of the kink chain). Above the HF-Bordoni peak, thermal activation leads to easy kink pair formation under the effect of the ultrasonic stress field, and the ultrasonic attenuation increases when the average displacement of the dislocation due to the low-frequency applied stress increases. The combination of the effect of the high- and low-frequency stresses allows one to simulate the experimental signatures. The excellent correspondence between the simulated and the experimental signatures (shape and evolution with the temperature) leads to the conclusion that the ultrasonic Bordoni peak is due to a KPF mechanism [18]. As a consequence, the Bordoni peak observed by internal friction measurements is due to a KPF mechanism. As in the case of the other fcc metals, one obtains a Peierls stress $\left(\sigma_{\mathrm{p}} \approx 10^{8} \mathrm{~N} / \mathrm{m}^{2}\right)$ greater than the critical resolved shear stress at $0 \mathrm{~K}\left(\sigma_{\mathrm{c}}(0) \approx 10^{6} \mathrm{~N} / \mathrm{m}^{2}\right)$.

\subsection{The softening process at low temperatures and the vacancy assisted KPF mechanism}

At very low temperatures, after a cold working and an annealing at $220 \mathrm{~K}$, the same signature shapes and evolution as those obtained at high temperatures were observed (Fig. 4B), but presenting a "mirror symmetry" at much lower temperature $(21 \mathrm{~K})$ [10]. The similarity of the signature shapes (parallelograms) leads to the conclusion that the dislocation-point defect interaction mechanism is also activated at very low temperatures. This fact is not surprising if one takes into account that the dislocationpoint defects interaction mechanism proposed in [18] is athermal. On the other hand, the similarity of the signature "mirror symmetry" observed at $21 \mathrm{~K}$ allows one to think that another KPF mechanism also appears at much lower temperature. The activation energy of this mechanism is of the order of $0.005 \mathrm{eV}$. This mechanism, which takes place at very low temperatures, would be the vacancy-assisted mechanism (short-circuit of the KPF mechanism) observed by Bujard and Quenet [11-13] in very pure aluminum samples after low temperature plastic deformation or irradiation.

In order to confirm that there is a lubrication process in lead, medium-frequency damping and modulus defect measurements were performed after cold working in lead samples. Figure 5 shows the results obtained after cold working at $24 \mathrm{~K}$ and for different annealing temperatures. A great similarity between these results in lead after cold working and those obtained in aluminum after cold working or irradiation has been observed [14-15]. Measurements after irradiation have not been performed in lead, but a perfect correlation between the annealing stages of the modulus after cold working and the annealing stages of the resistivity after irradiation can be seen in lead [10]. As a consequence, it can be concluded that the softening process in lead has to be attributed, as in aluminum, to a lubrication mechanism of the dislocation motion by vacancy-type intrinsic point defects. 
Moreover, just after cold working at $4 \mathrm{~K}$ (without annealing at $220 \mathrm{~K}$ ), other signatures (not reported here) have been observed, similar to those obtained by Bujard in very pure aluminum samples under the same conditions [12]. The shape of these signatures shares characteristics with the signatures observed in $\mathrm{Al}$ during the Bordoni relaxation, but in a much lower temperature range than that of the Bordoni relaxation, and these signatures disappear after annealing. Such an observation leads one to conclude that these signatures have the same origin as those discerned during the Bordoni relaxation (KPF mechanism), and that a short-circuit mechanism of the KPF is responsible for this kind of signature observed in lead and in aluminum just after cold working.

Recently, in her thesis, Quenet $[13,16]$ showed that these very low-temperature signatures can be well simulated numerically by a KPF model, using the same parameters as for the KPF signatures, but introducing a much smaller activation energy. Numerical simulations performed during this work in the case of lead were also in very good agreement with the experimental signatures [10].

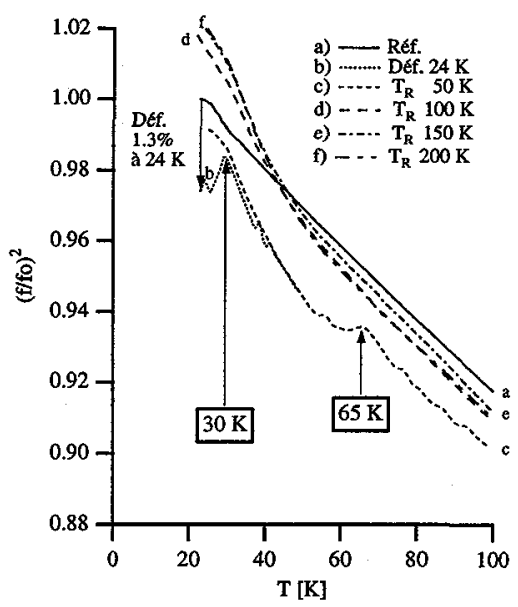

Figure 5: Modulus defect measurements as function of temperature in lead $5 \mathrm{~N}$ in the reference state (curve a), after a cold working of $1.3 \%$ at $24 \mathrm{~K}$ (curve b) and after different annealing temperatures (curves c-f). The reference frequency fo is $232 \mathrm{~Hz}$.

In summary, there is also a short-circuited KPF mechanism in lead, which is responsible for "KPF type" signatures observed by the coupling technique at very low temperatures just after cold working, and for "mirror-symmetry type" signatures observed at very low temperatures after cold working and annealing at $220 \mathrm{~K}$. This short-circuited KPF is also the mechanism responsible for the softening process observed in lead by internal friction measurements after cold working.

\section{CONCLUSION}

By internal friction and signature measurements in lead, two KPF mechanisms were observed. The first one, with an activation energy of $0.043 \mathrm{eV}$, is responsible for the low-frequency Bordoni peak relaxation $(40 \mathrm{~K})$ and for the new high-temperature signatures observed on each temperature side of the ultrasonic Bordoni peak $(150 \mathrm{~K})$. The second one, with an activation energy of $0.005 \mathrm{eV}$, corresponds to a shortcircuited KPF mechanism (vacancy assisted KPF), and it is responsible for the modulus softening effect and the signatures observed at very low temperatures after cold working. These results confirm the existence of the dislocation lubrication process recently proposed to explain the discrepancy between the low-temperature behaviour of the critical resolved shear stress in fcc metals and the mechanical spectroscopy measurements.

\section{Acknowledgments}

This work was partially supported by the Swiss National Science Foundation.

\section{References}

[1]. G. Fantozzi, I. G. Ritchie, J. Phys. 42 Colloque, C5 3-23 (1981).

[2]. G. Fantozzi, C. Esnouf, W. Benoit, I. G. Ritchie, Prog. Mat. Sc. 27, 311-451 (1982).

[3]. W. Benoit, M. Bujard, G. Gremaud, Phys. Stat. Sol. A 104, 427-431 (1987).

[4]. J. Baur, A. Kulik, J. Appl. Phys. 58, 1489-1492 (1985).

[5]. G. Gremaud, M. Bujard, W. Benoit, J. Phys. 61, 1795-1805 (1987).

[6]. W. P. Mason, J. Acoust. Soc. Am. 27, 643-653 (1955).

[7]. D. H. Niblett, J. Wilks, J. Phil. Mag. 1, 415-418 (1956).

[8]. A. Seeger, Phil. Mag. 1, 651-662 (1956).

[9]. A. Seeger, J. Phys. 32, C2 193-200 (1971).

[10]. O. Progin, Thèse n' 1436, EPFL, Lausanne Switzerland (1985).

[11]. M. Bujard, G. Gremaud, W. Benoit, J. Appl. Phys. 62, 3173-3183 (1987).

[12]. M. Bujard, Thèse $n^{\circ} 585$, EPFL, Lausanne Switzerland (1985).

[13]. B. Quenet, Thèse ${ }^{\circ}$ 990, EPFL, Lausanne Switzerland (1992).

[14]. J. Lauzier, J. Hillairet, A. Vieux-Champagne, W. Benoit, G. Gremaud, J. Phys.: Condens. Matter 1, 9273-9282 (1989).

[15]. J. Lauzier, J. Hillairet, G. Gremaud, W. Benoit, J. Phys.: Condens. Matter 2, 9247-9256 (1990).

[16]. W. Benoit, G. Gremaud, B. Quenet, Mater. Sci. Eng. A164, 42-57 (1993).

[17]. D. Wagner, F. Stangler, Phys. Stat. Sol. 41, k137-k140 (1977).

[18]. O. Progin, G. Gremaud, W. Benoit, "A model for the signatures observed in $5 \mathrm{~N}$ lead by two waves acoustic coupling method", in these proceedings 